\title{
Where the Wild Young M Dwarfs Are: the SUPERBLINK Proper Motion Survey and a Search for Low-mass Moving Group Candidates
}

\author{
Sébastien Lépine ${ }^{1}$ \\ ${ }^{1}$ Department of Physics and Astronomy, Georgia State University \\ email: slepine@astro.gsu.edu
}

\begin{abstract}
The SUPERBLINK survey catalogs all stars brighter than $R=19$ mag and with proper motions larger than 40 mas $\mathrm{yr}^{-1}$, down to a declination of $-33^{\circ}$. The catalog inevitably includes a significant fraction of the presumed low-mass members of several nearby young moving groups (Beta Pic, AB Dor, Tuc-Hor, Argus), or low-mass escapees from the Hyades and Pleiades clusters. We discuss opportunities and challenges in identifying the missing $\mathrm{M}$ dwarf members of these moving groups. While rounding up the majority of the potential $\mathrm{M}$ dwarf members of these groups, such samples are significantly affected by co-moving field stars, both young and old, due to the heavy clumping of the local field population in velocity space.
\end{abstract}

Keywords. surveys, stars: kinematics, stars: low-mass, brown dwarfs

\section{Searching for Young $M$ dwarfs}

The SUPERBLINK survey, is an all-sky census of $\sim 3$ million stars with very large

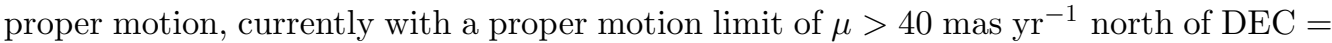

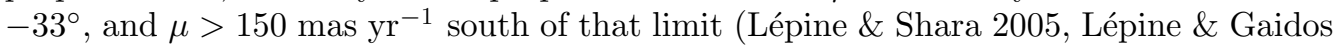
2011). The survey reaches optical magnitude $R<19 \mathrm{mag}$, has a high completeness rate within the proper motion and magnitude limits, and also a very high $(>99.5 \%)$ reliability, i.e. it is a 'verified' catalog, with virtually no spurious entries. The survey is accurately cross-matched to several photometric catalogs (ROSAT, GALEX, USNO-B1.0, SDSS, 2MASS). From a combination of proper motion and magnitude cuts, 233,727 M dwarfs estimated to be located within 100 parsecs of the Sun have been identified and their kinematics estimated (Fig. 1).

A kinematic search for young $M$ dwarfs can be performed by searching for stars that appear to be co-moving with local clusters or moving groups. The search also takes the magnitude and colors of the stars into account, searching for objects that are consistently over-luminous, like the known nearby young stars (see e.g. Lépine \& Simon 2009). The clear identification of hundreds of $\mathrm{M}$ dwarfs in the Pleiades and Hyades clusters show the method to be very efficient at recovering potential $M$ dwarf members (Fig. 2), but the contamination from field stars is severe, especially for nearby groups that are significantly spread out. This happens because the nearby young clusters and moving groups occupy a heavily populated region in velocity space, and are coincidentally co-moving with numerous local field stars, old and young.

In the end the method most probably round up a significant fraction of the M dwarf members of those young moving groups and clusters, but various follow-up observations are required to search for signs of youth and confirm group membership. 


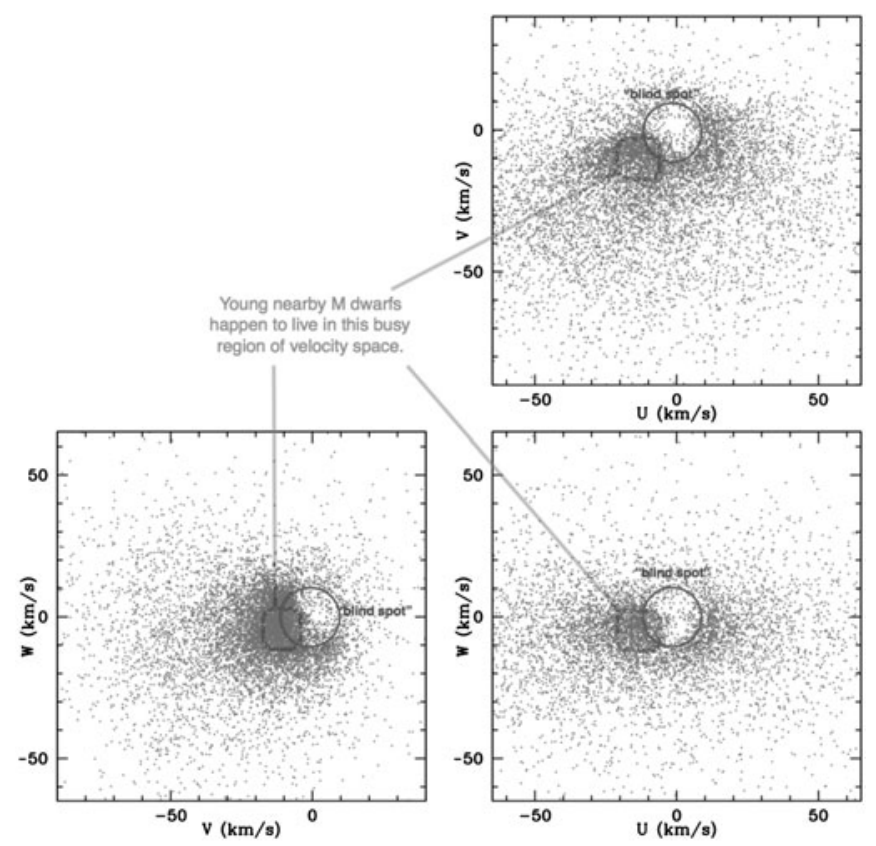

Figure 1. Kinematics from proper motion and photometric distances reveals that the velocity-space distribution of $\mathrm{M}$ dwarfs is very anisotropic and inhomogenous, consistent with the very same substructure or 'streams' observed for more massive G stars (Nordström et al. 2004).
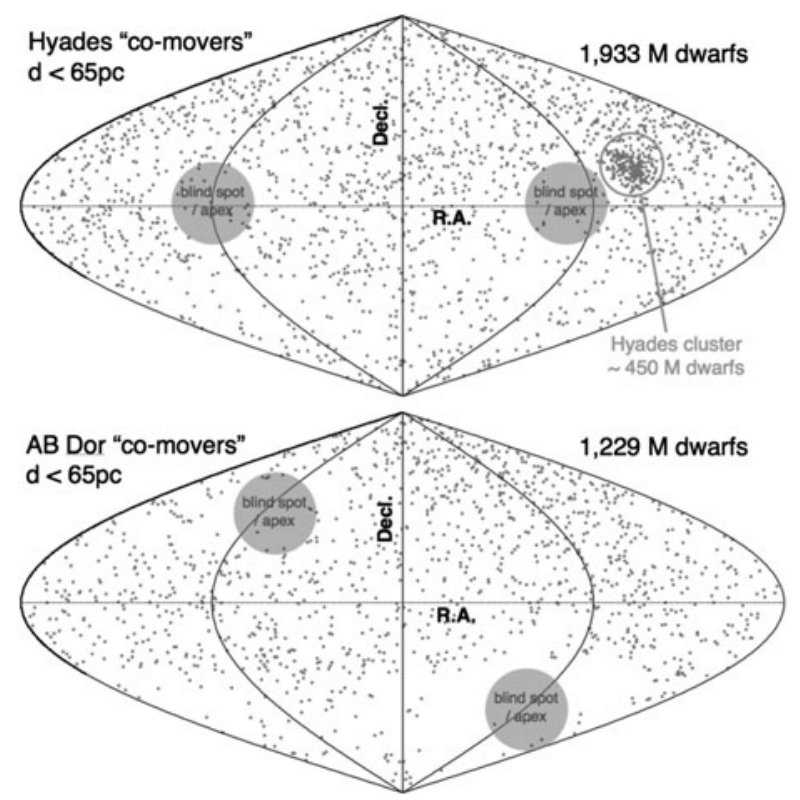

Figure 2. Kinematic selection: candidate stars from the Hyades and AB Dor moving group.

\section{References}

Lépine, S. \& Shara, M. M. 2005, AJ, 129, 13

Lépine, S. \& Gaidos, E. 2011, AJ, 142, 138

Lépine, S. \& Simon, M. 2009, AJ, 137, 383

Nordström, B., et al. 2004, A\&\&A, 418, 989 